\title{
Interhemispheric Binding of Ambiguous Visual Motion Is Associated with Changes in Beta Oscillatory Activity but Not with Gamma Range Synchrony
}

\author{
Gabriel Nascimento Costa ${ }^{1}$, João Valente Duarte ${ }^{1}$, Ricardo Martins ${ }^{1}$, \\ Michael Wibral ${ }^{2}$, and Miguel Castelo-Branco ${ }^{1}$
}

\begin{abstract}
In vision, perceptual features are processed in several regions distributed across the brain. Yet, the brain achieves a coherent perception of visual scenes and objects through integration of these features, which are encoded in spatially segregated brain areas. How the brain seamlessly achieves this accurate integration is currently unknown and is referred to as the "binding problem." Among the proposed mechanisms meant to resolve the binding problem, the binding-by-synchrony hypothesis proposes that binding is carried out by the synchronization of distant neuronal assemblies. This study aimed at providing a critical test to the binding-by-synchrony hypothesis by evaluating long-range con-
\end{abstract}

\section{INTRODUCTION}

The processing of sensory information is highly segregated across the human brain. In the visual cortex, this segregation is particularly striking owing to the distributed parallel processing of different perceptual features (e.g., shape, motion). Such functional organization raises the question of how the brain correctly integrates features of distinct objects perceived simultaneously during conscious experience. This became known as the binding problem (Von der Malsburg, 1981). One controversial hypothesis, known as the temporal binding or bindingby-synchrony (BBS) hypothesis, proposes that neurons coding for distinct features of an object fire synchronously, providing a temporal binding mechanism that reinforces the signaling of a select group of neurons while segregating others that do not stand for features of the same object (Singer \& Gray, 1995).

The BBS is supported by findings of synchrony between distant neuronal assemblies correlating with holistic perception (reviewed in Uhlhaas et al., 2009; Gray, 1999). In paradigms requiring interhemispheric auditory and visual integration, synchrony in the beta and gamma range has been found to predict perception (Hipp, Engel,

\footnotetext{
${ }^{1}$ University of Coimbra, Coimbra, Portugal, ${ }^{2}$ Goethe University,
} Frankfurt, Germany nectivity using EEG during a motion integration visual task that entails binding across hemispheres. Our results show that largescale perceptual binding is not associated with long-range interhemispheric gamma synchrony. However, distinct perceptual interpretations were found to correlate with changes in beta power. Increased beta activity was observed during binding under ambiguous conditions and originates mainly from parietal regions. These findings reveal that the visual experience of binding can be identified by distinct signatures of oscillatory activity, regardless of long-range gamma synchrony, suggesting that such type of synchrony does not underlie perceptual binding.

\& Siegel, 2011; Rose \& Buchel, 2005), and perceptual binding can be influenced by uncoupling oscillatory activity using antiphasic transcranial alternating current neurostimulation (tACS; Struber, Rach, Trautmann-Lengsfeld, Engel, \& Herrmann, 2014). Nonetheless, despite compelling evidence suggesting that dynamic neural assemblies can arise from the synchronized firing of neurons, the question of whether integration of visual features, that is, visual binding, relies on this synchronous activity remains controversial (Shadlen \& Movshon, 1999). Recent accounts on the functional relevance of synchronization phenomena emphasize that neural activity concentrated on particular phases of oscillatory cycles provides potential information encoding mechanisms (Goldfarb \& Treisman, 2013; Nikolic, Fries, \& Singer, 2013; Fries, 2009), but other findings point toward neurons carrying information by the linear integration of inputs, regardless of synchrony (Histed \& Maunsell, 2014; Kiani, Churchland, \& Shadlen, 2013).

To study long-range integration in perceptual binding, we took advantage of a previously described bistable stimulus that critically engages both visual hemifields and, consequently, requires integration across both visual hemispheres to form a coherent percept (Wuerger, Shapley, \& Rubin, 1996; Wallach, 1935). The percepts elicited by this ambiguous moving stimulus closely resemble the pattern and transparent motion of drifting plaids (formed by 
superimposed gratings), differing in that the gratings are presented in a nonoverlapping configuration. The "roofshaped" stimulus can thus be perceived as a single unified pattern moving downward or as two surfaces describing a horizontal movement inward, accompanied by the striking perception of being segregated across hemispheres. These configurations will be henceforth referred to as "bound" and "unbound" percepts, respectively. Using EEG, large-scale interhemispheric synchrony of oscillatory activity was measured while participants reported seeing the ambiguous image in its bound or unbound configurations. This simple paradigm offers an ideal setting for a critical test to the BBS, as it implies large-scale recruitment of neuronal populations with similar response properties that engage in integration, that is, binding, or segmentation of the moving stimulus. We found that interhemispheric synchrony in gamma band did not differ between different perceptual interpretations of the stimulus, contrary to the prediction of BBS that perceptual binding across the vertical meridian would entail interhemispheric synchrony. On the other hand, spectral signatures of increased power at beta frequencies were found to correlate with the perception of the bound stimulus. When the bound configuration was perceived, beta activity was increased compared with the unbound perception, with greater differences found over parietal regions. This suggests that the ambiguity might be resolved at hierarchically higher cortical areas without the need for synchronous activity at lower visual ones.

\section{METHODS}

\section{Participants}

Twenty-three healthy participants (12 women, 22-36 years old) were recruited from the student population and staff of the University of Coimbra. One participant is an author of this study. Thirteen participants performed both the ambiguous (main task) and unambiguous (control) tasks, eight performed exclusively the main ambiguous task, and two participants performed only the unambiguous control task. All participants had normal or corrected-to-normal vision and were right-handed. All procedures were conducted in accordance with the Declaration of Helsinki and approved by the ethics committee of the Faculty of Medicine of the University of Coimbra. Participants gave their informed written consent before the experiments.

\section{Stimulus}

Stimulus' properties were as follows: contrast $=100 \%$, duty cycle $=6 \%$, spatial frequency $=0.6$ cycle $/$ degree, orientation $=45^{\circ}$ relative to $x$ axis (left-side image), motion speed $=5^{\circ} / \mathrm{sec}$, stimulus size $=10^{\circ} \times 11^{\circ}($ vertical $\times$ horizontal), and viewing distance $=70 \mathrm{~cm}$, reproducing the stimulus first described by Hans Wallach (1935). A central blue cross (visual angle $=0.4^{\circ}$ ) was present as a fixation target at the visual midline to avoid gaze drift. A fully unambiguous control stimulus was created by displaying on top of the lines randomly distributed gray dots (600 dots; contrast $=10 \%$, visual angle $=0.2^{\circ} ;$ Figure $\left.1 \mathrm{C}\right)$, moving at the same speed as the grating pattern either descending or moving inward. Stimuli were generated in MATLAB (The MathWorks, Inc., Natick, MA) using the Psychophysics Toolbox (Brainard, 1997) and presented on an LCD monitor with a refresh rate of $60 \mathrm{~Hz}$.

\section{Experiment}

Participants continuously observed the ambiguous moving stimulus for $3 \mathrm{~min}$, while maintaining fixation at the central cross and reporting the perceived direction of motion by pressing and holding one button for downward motion/bound perception and another for inward motion/unbound perception. Participants could abstain from reporting either of the two percepts in case of doubt (i.e., by releasing both buttons, less than $2 \%$ of the total duration of the experiment). Although other alternative percepts were compatible with the ambiguous stimulus (e.g., orthogonal motion toward the center; half of the image moving vertically downward and the other moving horizontally inward), these were seldom reported by participants. At any rate, for the purposes of this study, these alternative configurations could be safely categorized as "unbound" because a separation of both halves of the image was a common feature to all and easily identifiable as such by the participants. For the unambiguous task, four runs were performed, lasting $3 \mathrm{~min}$, with the direction of the disambiguating moving dots set to change randomly every $2-3.5 \mathrm{sec}$ and perceptual reports performed as in the ambiguous task.

\section{Behavioral Analysis and Perceptual Dynamics}

The durations of each percept were binned to an adequate time window (2000 msec for the bound percept and $500 \mathrm{msec}$ for the unbound percept, based on average perceptual durations), and their distribution was fitted with a gamma distribution. The maximum likelihood estimates of $\alpha$ and $\beta$ parameters of a gamma distribution were calculated for each participant, and the deviation from the goodness of fit was assessed using the KolmogorovSmirnov test $(p>.05$; see Figure 1D).

\section{EEG Acquisition and Preprocessing}

EEG data were recorded using a 64-channel system (EasyCap, Munich, Germany) with $58 \mathrm{Ag} / \mathrm{AgCl}$ electrodes positioned according to the extended 10-20 system and four additional ones in a bipolar montage for vertical and 
horizontal EOG. Vertex referenced data were acquired continuously during each run. The signal was amplified, low-pass filtered at $200 \mathrm{~Hz}$, and digitized at $1000 \mathrm{~Hz}$. Electrode impedances were kept below $10 \mathrm{k} \Omega$ during acquisition and checked halfway through the session. EEG recordings were analyzed offline using MATLAB and EEGLAB (Delorme \& Makeig, 2004). Data sets were bandpass filtered (1-100 Hz, finite impulse response filter), and periods containing artifacts (e.g., swallowing, electrode pops) were removed by visual inspection. Blinks, eye movements, and other artifacts were removed by discarding epochs with amplitude exceeding $\pm 100 \mu \mathrm{V}$. In case bad channels were present, these were interpolated using spherical spline interpolation. Independent component analysis was computed, and components corresponding to muscle artifacts, identified by their scalp topography and spectra, were removed. A notch filter was applied at $50 \mathrm{~Hz}$ to eliminate powerline noise. Data were rereferenced to the average of all scalp electrodes. Surface Laplacian, when applied to the EEG signal, was computed using the Fieldtrip toolbox (Oostenveld, Fries, Maris, \& Schoffelen, 2011; www.ru.nl/neuroimaging/fieldtrip) and the spline interpolation method (Perrin, Pernier, Bertrand, \& Echallier, 1989, 1990).

\section{Epoch Selection}

Frequency analysis and connectivity estimates were carried out in epochs of stable perceptual states, that is, in between perceptual switches, as well as in epochs consisting of perceptual transitions. Epochs of stable bound or unbound perception, as reported by the participants, were obtained from the continuous EEG recording and segmented into nonoverlapping 1-sec epochs. The $250 \mathrm{msec}$ immediately preceding and following a button press were excluded from such analysis. Matching of the number of trials between conditions had to be carried out, because, for all participants, the bound/downward-moving percept was dominant $(\approx 75 \%$ of the total viewing time; see Figure 1E). A balanced analysis was performed by randomly selecting an amount of "bound" epochs equalling that of "unbound" for each participant (mean $=164.8$ epochs per participant, $S D=103.3$ epochs per participant). This selection was performed for each run so as not to bias the pooling of epochs from runs where one condition was underrepresented. Epochs of perceptual transition were defined as a time window of $\pm 2000 \mathrm{msec}$ around the button press reporting a change in the perceived configuration and divided as transitions from unboundto-bound and bound-to-unbound, where "0" represents the moment of motor report. Epochs that corresponded to or that followed a period of brief perceptual transition, considered as lasting less than 1500 msec (i.e., signalled by a button press of either perceptual configuration $\pm 1500 \mathrm{msec}$ around "0"), were excluded, as were epochs containing blinks. The number of epochs of perceptual transition was also matched between conditions. Five participants were excluded from this analysis of perceptual transitions because of a low number of resulting epochs (less than 30 for either condition; mean $=57.1$ epochs per participant, $S D=23.1$ epochs per participant).

\section{Frequency Analysis}

The power spectrum was calculated for stable epochs of each perceptual condition using Welch's modified periodogram method: Epochs were divided into overlapping segments, Hanning windowed, and transformed using a zero-padded fast Fourier transform. To assess event-related spectral changes, time-frequency analysis was performed on epochs of perceptual transition using an adaptive complex Morlet wavelet (three cycles for $4 \mathrm{~Hz}$ up to 33 cycles for $90 \mathrm{~Hz}$ ). Spectra obtained by these methods were used for the estimation of sensor level synchrony (see next section).

\section{Sensor Level Connectivity Analysis}

Synchrony between pairs of electrodes was estimated using imaginary coherence (ImCoh; Nolte et al., 2004) and phase coherence (Coh; Delorme \& Makeig, 2004). ImCoh was calculated for frequency $f$ as follows:

$$
\begin{aligned}
& \operatorname{ImCoh}_{\mathrm{ab}}(f)= \\
& \left|\operatorname{imag}\left(\frac{1}{n} \sum_{t=1}^{n} \frac{F_{\mathrm{a}}(f, t) \cdot F_{\mathrm{b}}(f, t)^{*}}{\sqrt{\left[F_{\mathrm{a}}(f, t) \cdot F_{\mathrm{a}}(f, t) *\right] \cdot\left[F_{\mathrm{b}}(f, t) \cdot F_{\mathrm{b}}(f, t)^{*}\right]}}\right)\right|
\end{aligned}
$$

where $F_{\mathrm{a}}(f, t)$ and $F_{\mathrm{b}}(f, t)$ are the spectral estimates of channels a and $\mathrm{b}$, respectively, for frequency $f$ and time point $t$ and $F_{\mathrm{a}}(f, t)^{*}$ and $F_{\mathrm{b}}(f, t)^{*}$ are their complex conjugates. The normalized cross-spectrum (coherency) was averaged over time (Cohen, 2014), and the modulus of its imaginary part was taken.

Coh was estimated as:

$$
\operatorname{Coh}_{\mathrm{ab}}(f)=\left|\frac{1}{n} \sum_{t=1}^{n} \frac{F_{\mathrm{a}}(f, t) \cdot F_{\mathrm{b}}(f, t)^{*}}{\left|F_{\mathrm{a}}(f, t) \cdot F_{\mathrm{b}}(f, t)^{*}\right|}\right|
$$

ImCoh and Coh were averaged over trials to estimate the average synchrony during each perceptual condition. ImCoh and Coh were estimated for all electrode pairs (see Figure 4). Planned comparisons of average synchrony related to perception were carried out between symmetrical clusters, namely, parietal (left: CP1, CP3, P1, and P3; right: $\mathrm{CP} 2, \mathrm{CP} 4, \mathrm{P} 2$, and $\mathrm{P} 4$ ), occipitotemporal (left: TP7, CP5, P7, P5, and PO7; right: CP6, TP8, P6, P8, and PO8), and occipital (left: $\mathrm{PO} 3$ and O1; right: $\mathrm{PO} 4$ and $\mathrm{O} 2$ ). For estimating synchrony during perceptual transitions, ImCoh and Coh were computed using sliding time segments (200-msec window) and averaged across trials. 
Granger causality (GC) was computed for all electrode pairs using a nonparametric bivariate spectral matrix factorization method (Dhamala, Rangarajan, \& Ding, 2008) using Fieldtrip. GC exerted from electrodes located close to visual areas of one hemisphere over the other (right hemisphere: CP2, CP4, CP6, TP8, P2, P4, P6, P8, PO4, PO8, and O2; left hemisphere: CP1, CP3, CP5, TP7, P1, P3, P5, P7, PO3, PO7, and O1) was averaged for all electrode pairs of interest and provided an estimate of connectivity across hemispheres.

\section{Source Level Connectivity Analysis}

Coherent activity between brain sources during bound/ unbound percepts was assessed using the Dynamic Imaging of Coherent Sources beamformer algorithm. Frequency analysis of each sensor was carried out using a multitaper method based on Slepian sequences for three frequency bands of interest (beta $=21 \pm 8 \mathrm{~Hz}$, low gam$\mathrm{ma}=38 \pm 7 \mathrm{~Hz}$, high gamma $=68 \pm 12 \mathrm{~Hz}$; the number of tapers was adjusted according to the intended spectral smoothing). The Fourier spectrum for each source was obtained by projecting the sensor level spectrum onto sources defined by a regular $8-\mathrm{mm}$ grid restricted to the gray matter (2527 sources), as defined by the segmentation of the Colin27 anatomical MRI (Montreal Neurological Institute; imaging.mrc-cbu.cam.ac.uk/imaging/ MniTalairach). Beamformer filters were computed on the combined data consisting of both conditions, and then, the common filter was applied to each condition individually. The cross-spectrum of each source pair was computed, and the connectivity was estimated as the phase-locking value (PLV), as it is readily implemented in Fieldtrip and comparable with Coh as computed in EEGLAB. A seed-based analysis of connectivity was carried out using a probabilistic map of the human visual cortex (scholar.princeton.edu/napl; Wang, Mruczek, Arcaro, \& Kastner, 2015). The presence of volume conduction effects between sources of interest was assessed with a shift test, using the Transfer Entropy toolbox (TRENTOOL; Lindner, Vicente, Priesemann, \& Wibral, 2011).

\section{Automatic Classification of Perceptual Changes}

Perceptual changes at trial level were classified as boundto-unbound or unbound-to-bound using Support Vector Machine (SVM; Cortes \& Vapnik, 1995) with a linear kernel model, based on the power spectrum over time. The perceptual change period was described using a set of 170 features (average power spectrum for two time bins, before and after a perceptual change $[-2000$ to 0 and 0-2000 msec, respectively]), and 85 frequencies (6-90 Hz). For each participant, an SVM classifier was trained and tested using a threefold cross-validation scheme with a balanced design. The average performance of the SVM classifier was evaluated using permutation tests based on 300 randomizations of trials selected for training and testing, compared against the performance of an analo- gous SVM classifier trained with random labels (Ojala \& Garriga, 2010).

\section{Statistical Analysis}

Statistical differences were analyzed using the nonparametric Wilcoxon signed rank test, paired by participant, unless otherwise stated. $p$ values are presented either uncorrected or adjusted for multiple comparisons using the false discovery rate (FDR; Benjamini \& Yekutieli, 2001). To test for statistically significant differences in timeresolved spectral power and control for multiple comparisons, time courses of oscillatory activity (i.e., power) were compared using a nonparametric cluster-based statistic (Maris \& Oostenveld, 2007), and critical $t$ values were selected using the Monte Carlo approach. Source level analysis was performed using cluster-based statistics, as implemented in Fieldtrip (Maris \& Oostenveld, 2007).

\section{RESULTS}

\section{Behavior}

The stimulus consisted of moving angled gratings mirrored at the vertical meridian of the visual field. Under continuous motion, the physically unchanged pattern yielded two alternative percepts: a single coherent pattern moving downward (Figure 1A) or two independent surfaces moving inward (Figure 1B). The latter perceptual state was commonly associated with reports of a virtual phase offset of the perceptually segregated lines between both halves of the image. The main perceptual task consisted of the continuous presentations of the ambiguous moving stimulus, during which participants were to report seeing a bound or unbound configuration by continuously pressing the corresponding key (one for the bound, another for the unbound configuration). The dynamics of bistability was studied based on the duration of the reported percepts. The durations of each percept followed a gamma distribution (16/21 participants for the unbound and 17 for the bound percept; KolmogorovSmirnov test, $p>.11$; see Figure 1D), which are typical of perceptual rivalry (Leopold \& Logothetis, 1999). Unlike other bistable stimuli such as the Necker cube, rivalry was asymmetrical and skewed toward a bound perception. The mean duration of the bound percept $(8.23 \pm 0.65 \mathrm{sec})$ was significantly longer than that of the unbound percept $(2.47 \pm 0.26 \mathrm{sec}$; Wilcoxon signed rank test, $p<.0001$; see Figure $1 \mathrm{E}$ ), resulting in an overall $77.4 \pm 2.2 \%$ of the total time spent perceiving a bound configuration. Moreover, similar to what is perceived with plaids (Hupe \& Rubin, 2003), the present stimulus was always first seen as a bound image, describing a descending global motion, and the duration of the first percept was consistently longer than subsequent ones: $14.58 \pm 1.53 \mathrm{sec}$ for the first bound percept compared with $7.64 \pm 0.58 \mathrm{sec}$ for the average of the following bound percepts $(p<.0001$; see Figure 1F). 

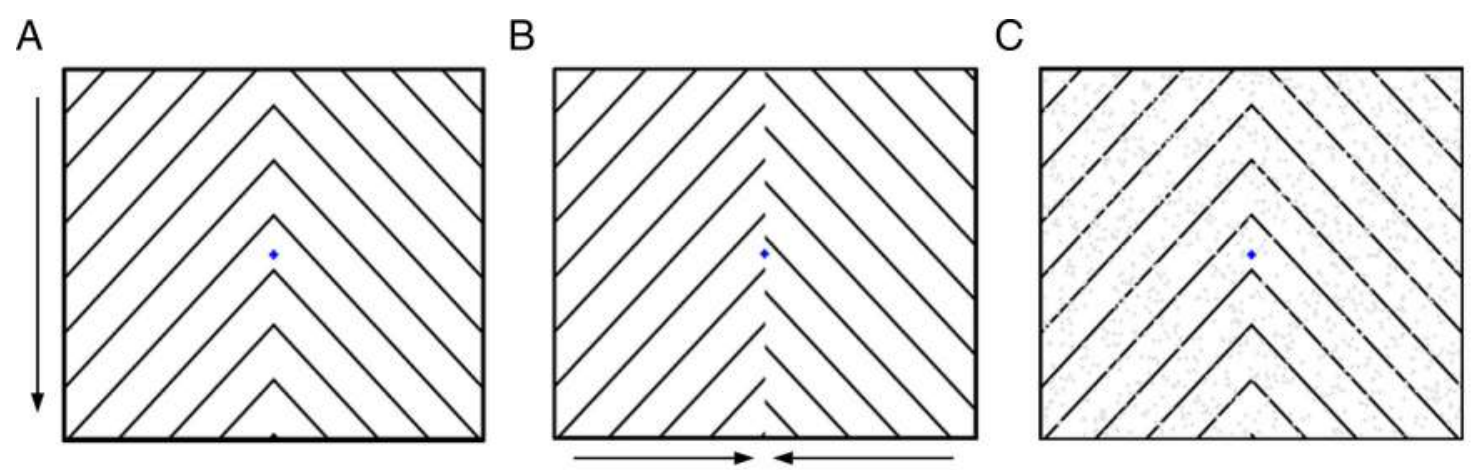

$\mathrm{D}$

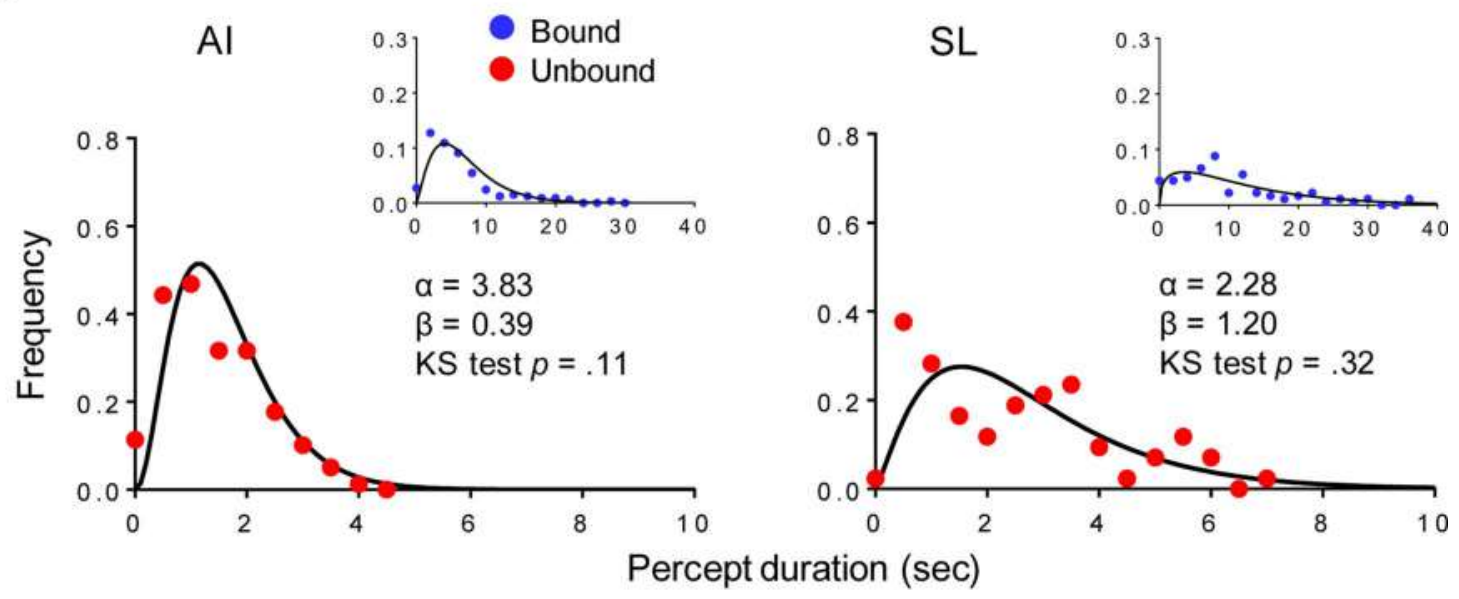

$E$

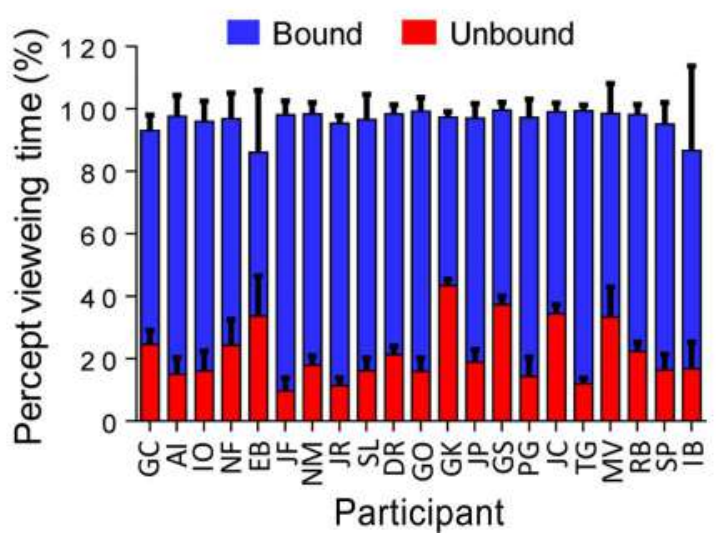

$\mathrm{F}$

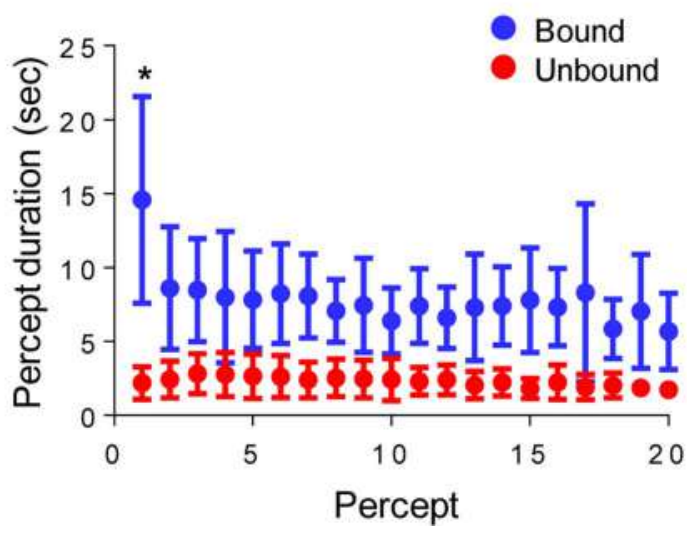

Figure 1. Roof-shaped moving stimulus and dynamics of perceptual bistability. Bistability resulting from continuous viewing of the ambiguous moving stimulus was characterized by alternating periods of interpreting the figure in its perceptually "bound" conformation (A), that is, both sides of the image being perceived as following the same vertical path, lines in phase, meeting at the center with no lag, and its "unbound" configuration (B) characterized by horizontal motion toward the center, an illusory border separating both sides and lines appearing, illusorily, to be out of phase. Arrows indicate the perceived direction of motion. (C) The unambiguous stimulus strongly induced perception of motion of the two sides of the image in the same direction as the moving dots. (D) Percept durations during ambiguous stimulation are adequately fit into a gamma distribution for 16 of 21 participants (black line, gamma fit; Kolmogorov-Smirnov test, $p>.05$; dots, histogram of percept duration). Best fit parameters and KolmogorovSmirnov test $p$ values are shown for the unbound percept for two participants. (E) Perceptual dominance during ambiguous stimulation for individual participants, measured as percentage of total reported perception (mean $\pm S D$ ). (F) Average duration of each percept (bound and unbound) during ambiguous stimulation as a function of its ordinal position. The first bound percept is significantly longer than subsequent ones (Wilcoxon signed rank test, $* p<.0001)$. Data are mean $\pm S D$.

An unambiguous stimulus, produced by overlaying dots moving in either a downward or an inward direction (Figure 1C), resulted in unequivocal bound or unbound percepts, that is, bound configuration when dots moved down- ward and unbound configuration when dots moved inward, which were readily reported as such by participants. Moreover, the perceptual properties of perceiving a bound or unbound percept (e.g., lines in or out of phase, disparity in 
depth perception) were reported by participants to be similar to the ones elicited by the ambiguous stimulus.

\section{Interhemispheric Coherence during Visual Binding}

The bistability resulting from the continuous viewing of this stimulus offers an opportunity to test whether perceptual binding, that is, under constant physical stimulation compatible with distinct percepts (bound as opposed to unbound), correlates with increased synchrony between distant neural assemblies. Importantly, in the current setting, the stimulus is observed across both visual hemifields, hence, according to the BBS, synchrony is expected to be increased over visual areas across hemispheres when the bound configuration is perceived. ImCoh, a connectivity measure that avoids confounds due to volume conduction by excluding zero-lag phase synchrony (Nolte et al., 2004), was determined for 1-sec-long EEG epochs consisting of stable bound and unbound percepts, according to participants' reports when viewing the ambiguous stimulus. During periods of stable perception, interhemispheric connectivity was found to be similar for both percepts, as ImCoh between the main posterior clusters studied-parietal, occipitotemporal, and occipital—of each hemisphere did not differ over a wide range of frequencies $\left(8-90 \mathrm{~Hz}, p>.2\right.$ for $\mathrm{ImCoh}_{\text {bound }}>$

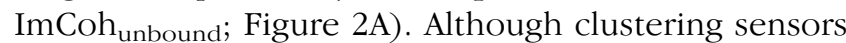
may be an adequate strategy to reduce the number of comparisons one has to carry out-connectivity measures can result in an overwhelming amount of data points, $1 / 2 \cdot N^{2}$ for $N$ electrodes - this strategy can also lead to a dilution of effects that might be observed over a small area covered by few electrodes. For this reason, we extended our analysis to differences over all pairs of electrodes, focusing on synchrony over beta and gamma bands, as the latter are emphasized in binding models (Singer, 2001) and the former are posited as the most suitable for longrange communication (Kopell, Ermentrout, Whittington,

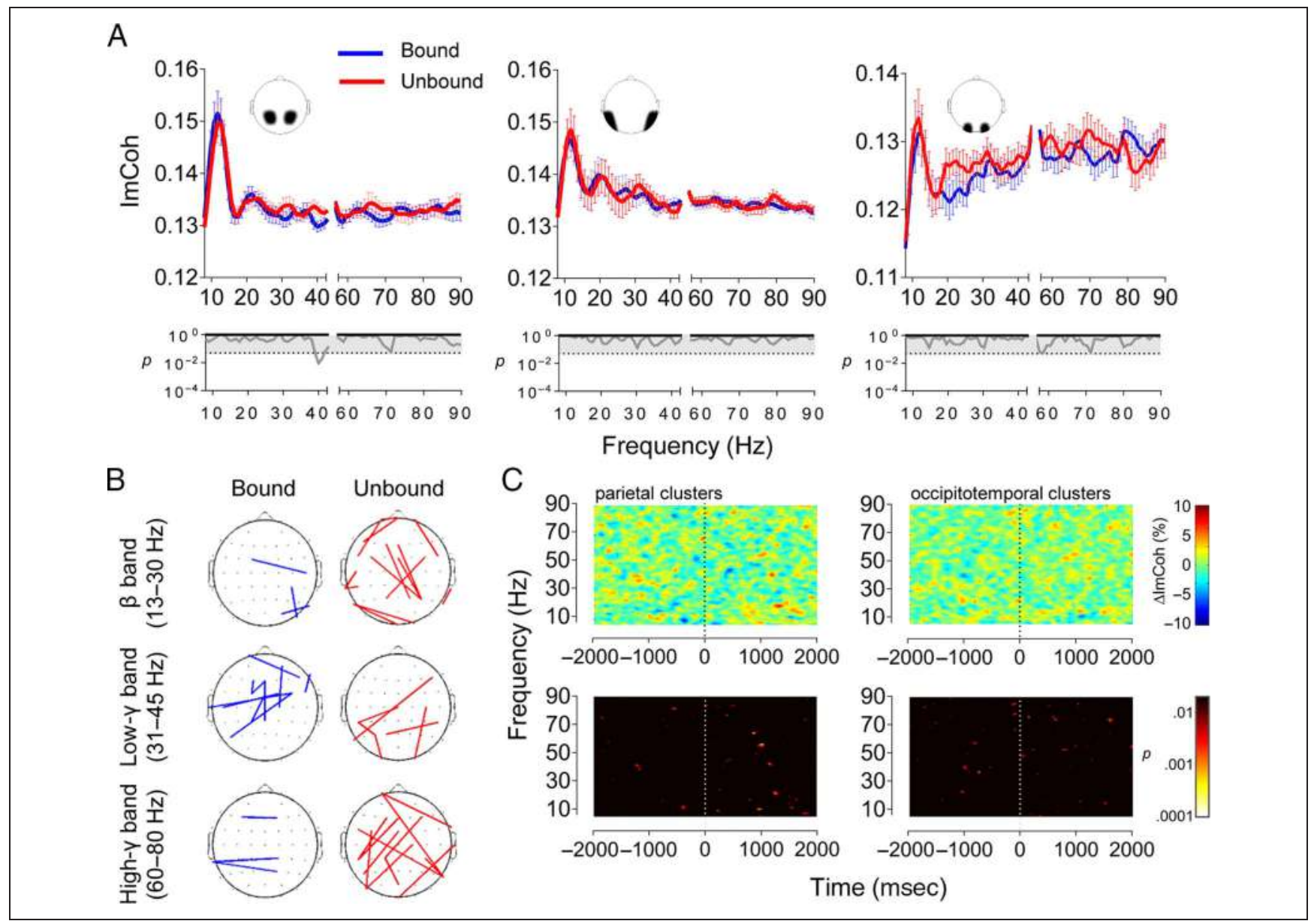

Figure 2. ImCoh across hemispheres during distinct perceptions under ambiguous conditions. (A) ImCoh between clusters of electrodes located at distinct hemispheres (parietal, left; occipitotemporal, center; and occipital, right; see Methods) was estimated for periods of stable bound and unbound perceptions. Data are mean $\pm S E M$. Bottom panels show uncorrected (Wilcoxon signed rank test, gray lines) and FDR-corrected (black lines) $p$ values. The shaded area depicts the region above the significance threshold $(p>.05)$. (B) ImCoh between all pairs of electrodes for beta and low- and high-gamma frequencies. Lines represent channel pairs with increased synchrony for either bound (blue) or unbound (red) perception, using a lenient threshold of $p<.01$ (uncorrected for multiple comparisons). (C) Time-frequency plots (top) of $\Delta \mathrm{ImCoh}$ (\% difference of ImCoh, "to-bound" minus "to-unbound" transitions) for parietal and occipitotemporal clusters. Lower plots show uncorrected $p$ values. 


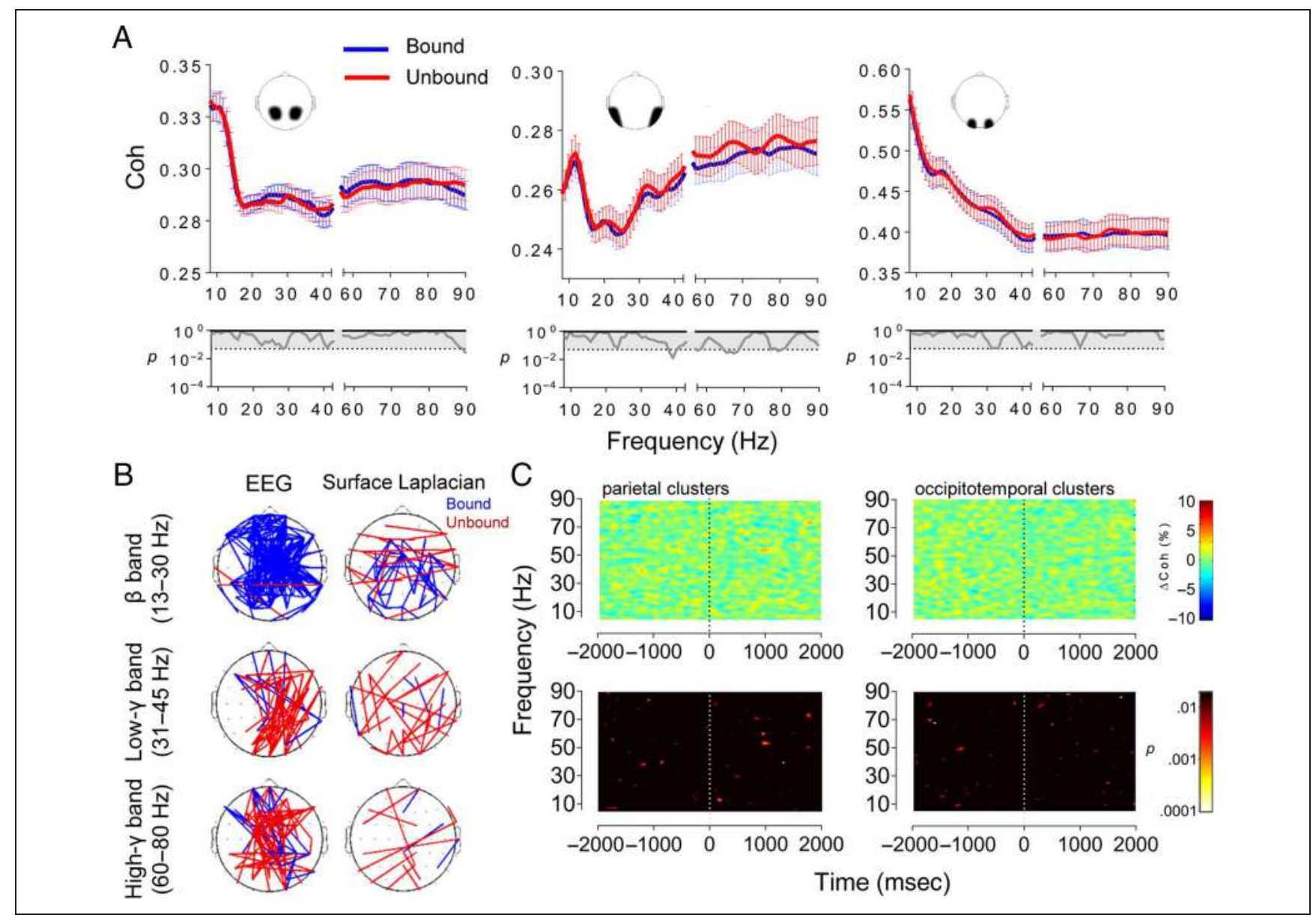

Figure 3. Interhemispheric Coh during distinct perceptions under ambiguous conditions. (A) Coh between clusters of electrodes located at distinct hemispheres (parietal, left; occipitotemporal, center; and occipital, right; see Methods) estimated and represented as in Figure 2. Bottom panels show uncorrected (Wilcoxon signed rank test, gray lines) and FDR-corrected (black lines) $p$ values. The shaded area depicts the region above the significance threshold $(p>.05)$. (B) Coh between all pairs of electrodes for beta and low- and high-gamma frequencies, for EEG (left) and surface Laplacian (right). Lines represent channel pairs with increased synchrony for either bound (blue) or unbound (red) perception, using a lenient threshold of $p<.01$ (uncorrected for multiple comparisons). (C) Time-frequency plots (top) of $\Delta$ Coh (\% difference of Coh, "to-bound" minus "to-unbound" transitions) for parietal and occipitotemporal clusters. Lower plots show uncorrected $p$ values.

\& Traub, 2000). Pairs of electrodes showing increased ImCoh during bound (blue lines) or unbound (red lines) periods are represented in Figure 2B. As can be seen, of the total number of electrode pairs, differences were found for a few ( $p<.01$, uncorrected), but no discerning pattern of interhemispheric synchrony is present at the sensor level. Of the pairs with significant differences, only few correspond to increased connectivity across hemispheres (1/3 electrode pairs for bound vs. 6/13 for unbound, for the beta band; $3 / 10$ vs. $2 / 6$, for low gamma; $3 / 3$ vs. $4 / 11$, for high gamma). An exhaustive analysis of all comparisons performed as shown in Figure $4 \mathrm{~A}$ also reveals little difference between the two percepts.

Despite these results not showing a positive association between visual binding and neural synchrony, one can conceive that binding might not rely on long periods of synchronous activity but on transient increases in synchrony. In that case, events of increased synchrony concentrated around the moment binding is realized would offer a better indication of its role in binding. With this in mind, we estimated ImCoh during epochs comprising events of perceptual transition. Figure $2 \mathrm{C}$ shows the difference in interhemispheric coherence between the two types of transitions, to bound or to unbound percepts, $\left(\Delta \mathrm{ImCoh}=\left[\mathrm{ImCoh}_{\mathrm{toB}}-\mathrm{ImCoh}_{\mathrm{toU}}\right] / \mathrm{ImCoh}_{\mathrm{toB}}\right)$ for two different clusters. Small changes $(<10 \%)$ can be seen across the spectrogram but are of brief duration $(<100 \mathrm{msec})$ and widely scattered, not offering compelling evidence of a change in synchrony related to a change in perceptual states.

Although spurious connectivity arising from volume conduction can be eliminated by only accounting for nonzero-lag phase synchrony, one risks ignoring a contribution of actual zero-lag phase synchrony to binding. In fact, early seminal work suggesting a temporal code for binding identified that zero-lag gamma synchrony correlated with the binding of visual features (Engel, Konig, Kreiter, \& Singer, 1991; Gray, Konig, Engel, \& Singer, 1989). For this reason, synchrony including zero-phase lag, as measured by Coh, was estimated for both perceptual conditions (Figure 3). As with ImCoh, interhemispheric Coh appears 
similar between posterior regions for both bound and unbound perceptions across several frequencies (Figure 3A). For specific frequency bands, increased Coh is observed for bound perception in the beta band (35/167 electrode pairs for bound vs. $2 / 2$ for unbound represent increased Coh between electrode pairs at opposing hemispheres; paired $t$ test, $p<.01$; Figure 3B, left), but this difference is absent from Coh estimated for the surface Laplacian (Figure 3B, right). The transformation to surface Laplacian was performed to increase topographical specificity and filter out spatially broad features resulting from volume conduction of scalp potentials. These results suggest a likely contribution by volume conduction to Coh estimates. Likewise, no increase in interhemispheric Coh is apparent at gamma frequencies (Figures 3B and 4B and $\mathrm{C}$ ). Considering the small differences and high $p$ values, one should be cautious in further interpreting these results as positive findings. Figure 3C shows that little difference in Coh is found also around the moment of perceptual switch. Similarly, for the unambiguous stimuli,

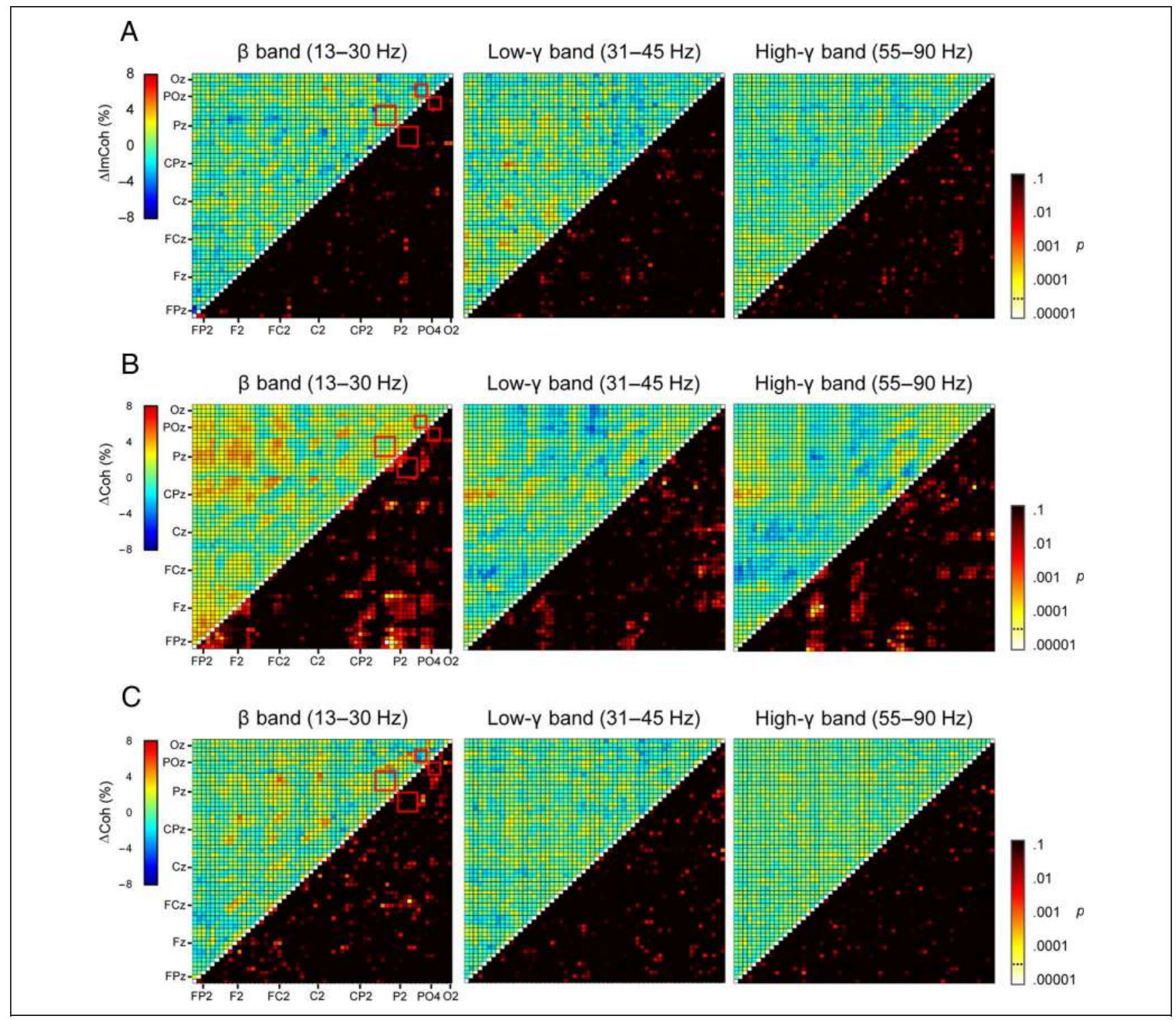

Figure 4. Connectivity differences between perceptual interpretations. An exhaustive analysis of ImCoh (A) and Coh (B, C) differences between bound and unbound ambiguous perceptions is displayed in the form of grid plots for three frequency bands (beta, low-gamma, and high-gamma frequencies). Each node represents the estimate of connectivity difference, bound minus unbound, for a pair of electrodes as depicted in the $x$ and $y$ axes. The top part represents percent difference of connectivity estimates (hot colors correspond to higher synchrony in bound, whereas cold colors correspond to higher synchrony in unbound), whereas the bottom half shows uncorrected $p$ values of the difference (dashed line represents Bonferroni-corrected $p<.05$ ). A depicts differences in ImCoh between bound and unbound percepts, whereas B and C represent differences in Coh for EEG and for surface Laplacian, respectively. Interhemispheric parietal and occipital electrode pairs where increased synchrony is expected to correlate with visual binding across hemifields according to the BBS are highlighted by red squares. Channel order in the $y$ axis is displayed from top to bottom as follows: O2, Oz, O1, PO8, PO4, POz, PO3, PO7, P8, P6, P4, P2, Pz, P1, P3, P5, P7, TP8, CP6, CP4, CP2, CPz, CP1, CP3, CP5, TP7, T8, C6, C4, C2, Cz, C1, C3, C5, T7, FT8, FC6, FC4, FC2, FCz, FC1, FC3, FC5, F8, F6, F4, F2, Fz, F1, F3, F5, F7, AF4, AF3, FP2, FPz, and FP1. 
Figure 5. GC and synchrony between cortical sources during distinct percepts under bistability. (A) GC was estimated for clusters of electrodes located at distinct hemispheres (left and right hemisphere posterior regions; see Methods) and is represented as the spectrum of mean GC from the left to right cluster and from the right to left cluster (mean \pm SEM). Bottom panels show uncorrected (Wilcoxon signed rank test, gray lines) and FDR-corrected (black lines) $p$ values. The shaded area depicts the region above the significance threshold $(p>$ $.05)$. (B) Matrices show differences in GC between bound and unbound percepts for three frequency bands (beta, low-gamma, and high-gamma frequencies) for posterior channel pairs (CP to O; order as in Figure 4). Channels in the $y$ axis Grangercause channels in the $x$ axis. Differences are represented as $z$ values of the comparison bound versus unbound, estimated using the Wilcoxon signed rank test (dashed lines on scale represent $z$ values with Bonferroni-corrected $p<.05$ ). Interhemispheric parietal and occipital electrode pairs are highlighted by red squares. (C) Connectivity analysis (PLV) using beamformer and gray-matter sources.

Projected $t$ values of synchrony difference (bound vs. unbound perception) in the beta frequency band between sources in seed areas (dark blue) and all other gray-matter sources. Synchrony was studied in early visual areas (V1) and extrastriate areas (V3, hMT/V5), identified using a probabilistic map of visual areas (see Methods). Sources that belong to clusters with significantly higher synchrony in the bound percept are represented by black dots in their original position $(p<.05$, permutation statistics; see source analysis methods).
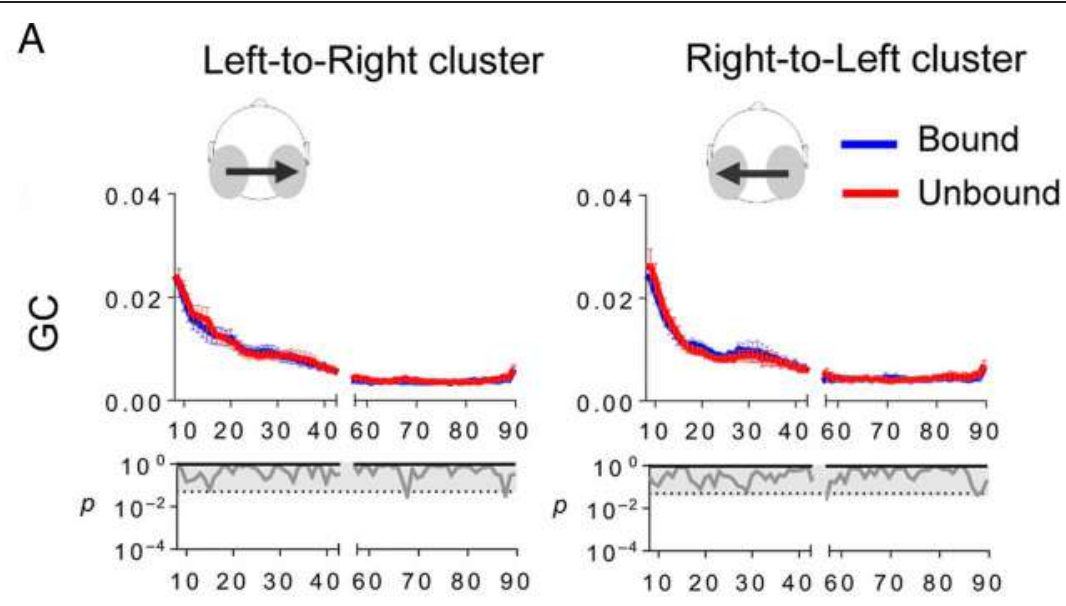

Frequency $(\mathrm{Hz})$

B

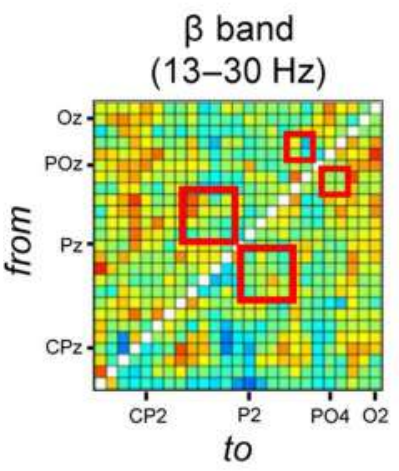

$$
\begin{aligned}
& \text { Low-y band } \\
& (31-45 \mathrm{~Hz})
\end{aligned}
$$

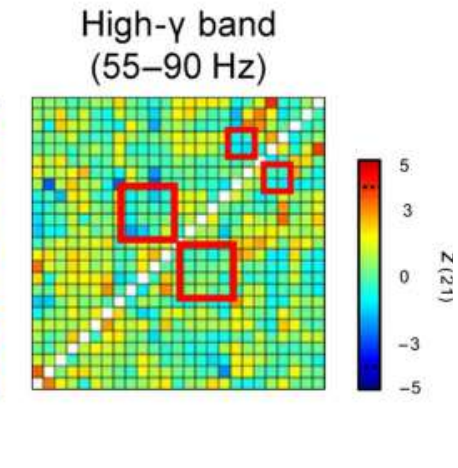

C
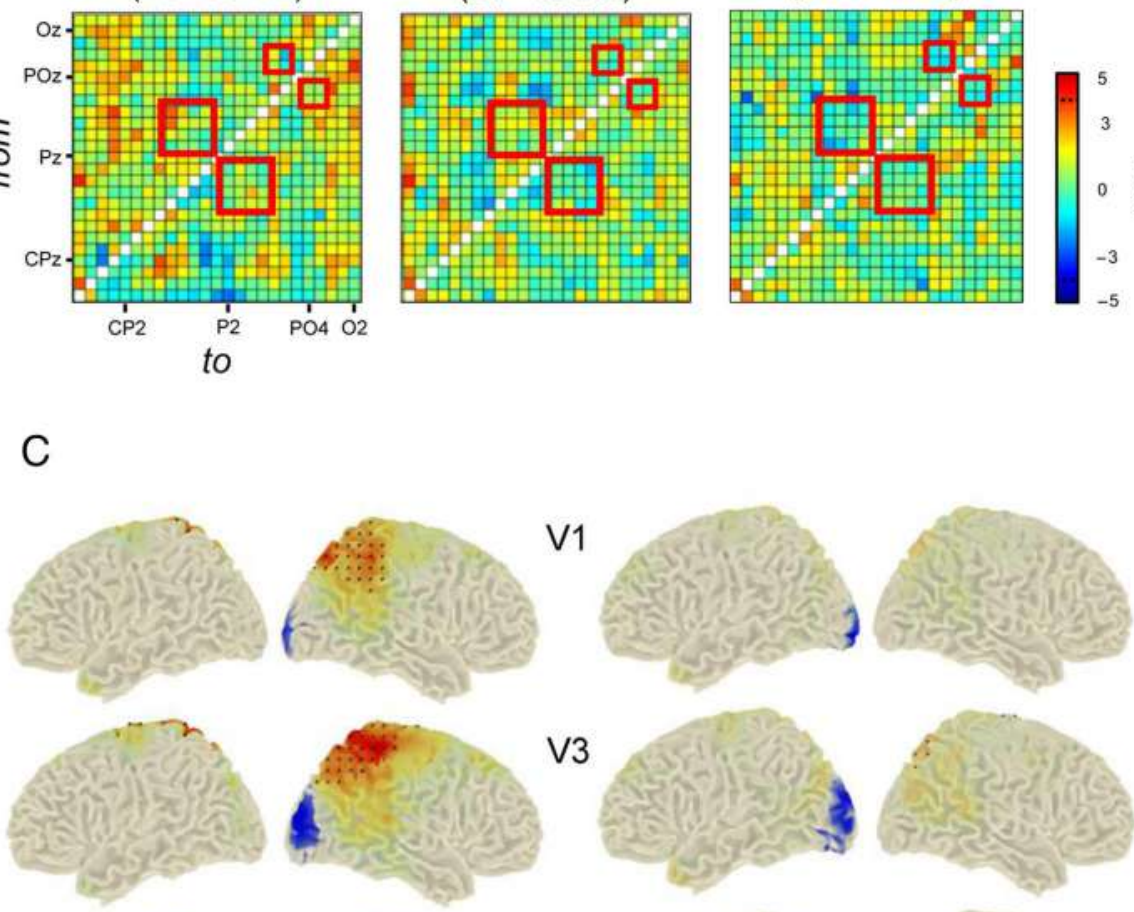

V3
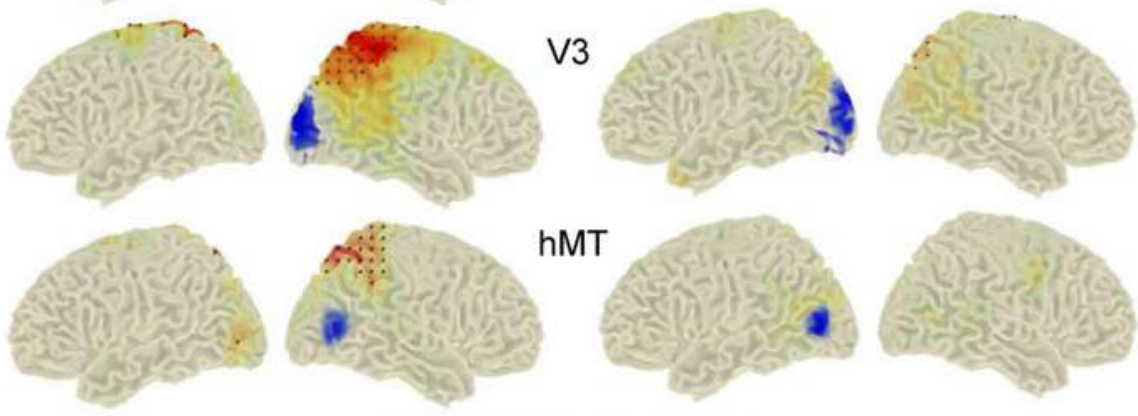

hMT
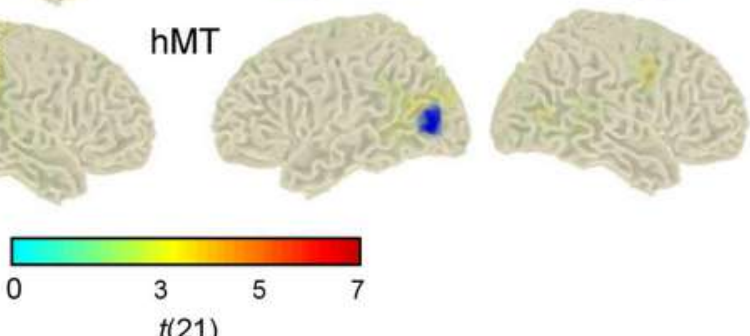

$t(21)$ interhemispheric Coh is either similar between the two percepts or absent in surface Laplacian (data not shown).

In addition, we studied connectivity across hemispheres by measuring directed influences between brain regions with GC. Information flow from one hemisphere to the other was similar between the two perceptual conditions across studied frequencies (Figure 5A and 5B). Although GC does not necessarily assess phase synchrony, in 
conditions of increased synchrony, GC should be higher as long as the influence of one signal over the other is not simultaneous. As can be seen in Figure 5B, this was not observed for either the bound or unbound percepts over visual areas for the main high-frequency bands studied $(>13 \mathrm{~Hz})$.

Finally, synchrony between cortical sources at the three frequency bands was studied using source reconstruction, which may be more appropriate than studying synchrony at the sensor level, with an adaptive linear spatial filtering, that is, beamformer (Gross et al., 2001). Seed-based differences in synchrony between the two percepts were estimated using three ROIs: early visual cortex (V1), extrastriate cortex (V3), and human middle temporal area (hMT/V5), as identified with a probabilistic map of visual areas (Wang et al., 2015). The difference in synchrony at beta frequencies associated with distinct percepts (Figure 5C), between the seeds (dark blue regions) and all gray-matter sources, shows that synchrony (as estimated with the PLV) is increased during bound perception, but this difference is mainly restricted to the right parietal cortex. Although a cluster of significant differences can be found in the contralateral hemisphere close to extrastriatal visual areas, including putative hMT + /V5 (Figure 5C, bottom left), this significant difference is of smaller magnitude, does not reach significance when the opposite region is analyzed (Figure 5C, bottom right), and appears to be affected by volume conduction (11/21 participants show instantaneous mixing between signals, as assessed with the shift test). No significant differences in synchrony were found for low- or highgamma frequencies (data not shown).

\section{Brain Oscillations Associated with Distinct Perception}

Phase-based methods of estimating connectivity are usually employed to study long-range communication, that is, communication between brain regions separated by more than $1 \mathrm{~cm}$, with conduction delays greater than 10 msec (Varela, Lachaux, Rodriguez, \& Martinerie, 2001). Nonetheless, the synchronous activity of neurons at the local level can lead to increased oscillatory power, by virtue of the summation of correlated neuronal activity (Musall, von Pfostl, Rauch, Logothetis, \& Whittingstall, 2014). These oscillations are modulated by perception and can reveal states of conscious visual experience in situations of ambiguity (Smith, Gosselin, \& Schyns, 2006; VanRullen, Reddy, \& Koch, 2006). We explored changes in the power of brain oscillations as signatures of visual perception. Eye movements were recorded during the

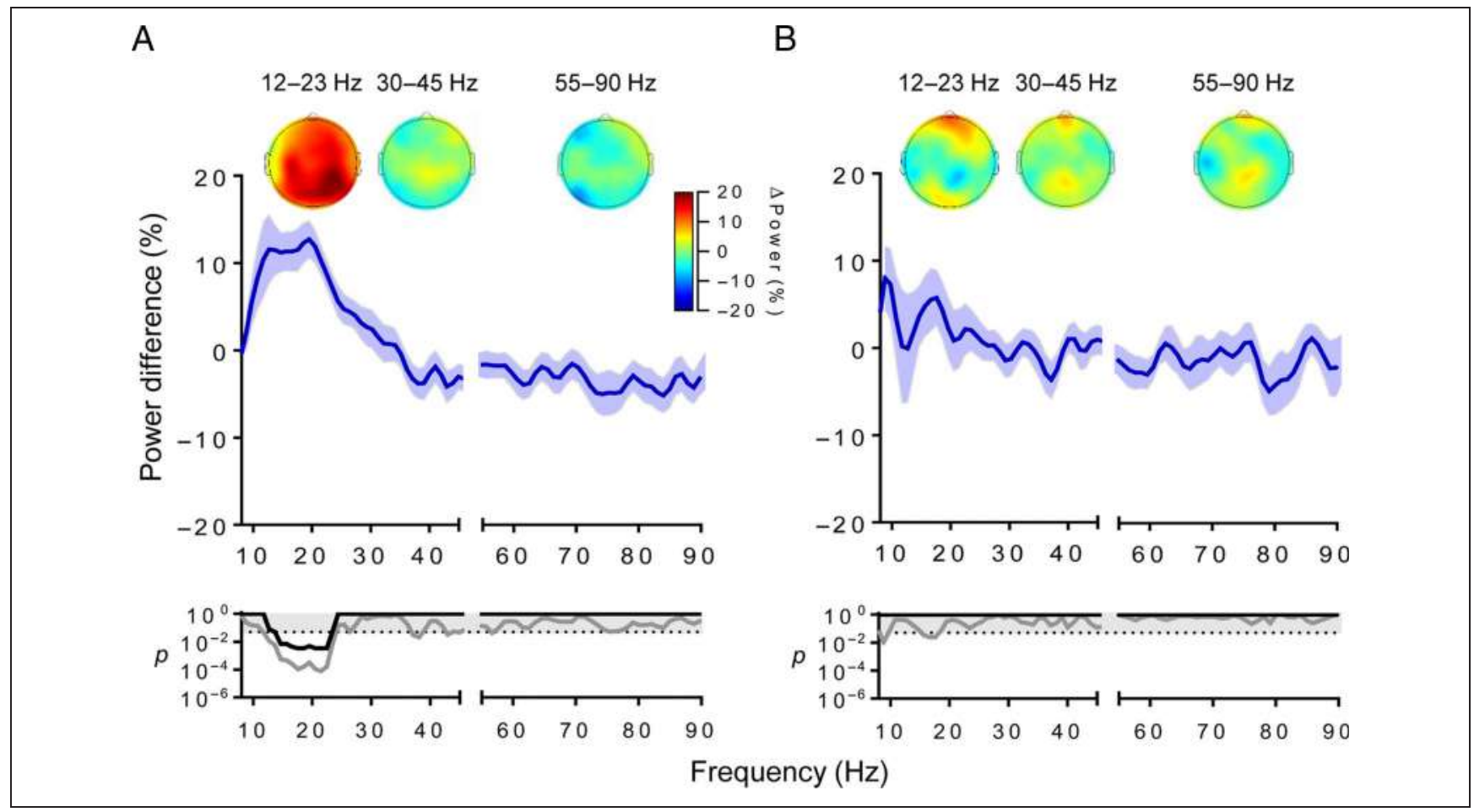

Figure 6. Global power differences are associated with distinct percepts. (A) Increased power in the alpha and beta frequency bands is associated with bound perception. Curves represent percentage of average power difference (bound - unbound) over all electrodes, estimated for stable periods. The shaded area represents SEM. Bottom panels show uncorrected $p$ values (gray lines) and FDR-adjusted $p$ values (black lines). The shaded area depicts the region above the significance threshold $(p>.05)$. Significant differences were found for a frequency band around $12-23 \mathrm{~Hz}$ $(p<.05$, corrected for multiple comparisons). The topographies of power difference (\%) are shown for three frequency bands. (B) Spectrum differences for the bound and unbound unambiguous conditions. No significant differences of global power were found between the two percepts. The increased alpha/beta activity over parietal areas found during bound perception for the ambiguous stimulus is also absent in the unambiguous stimulus. $p$ values are represented as in A. 

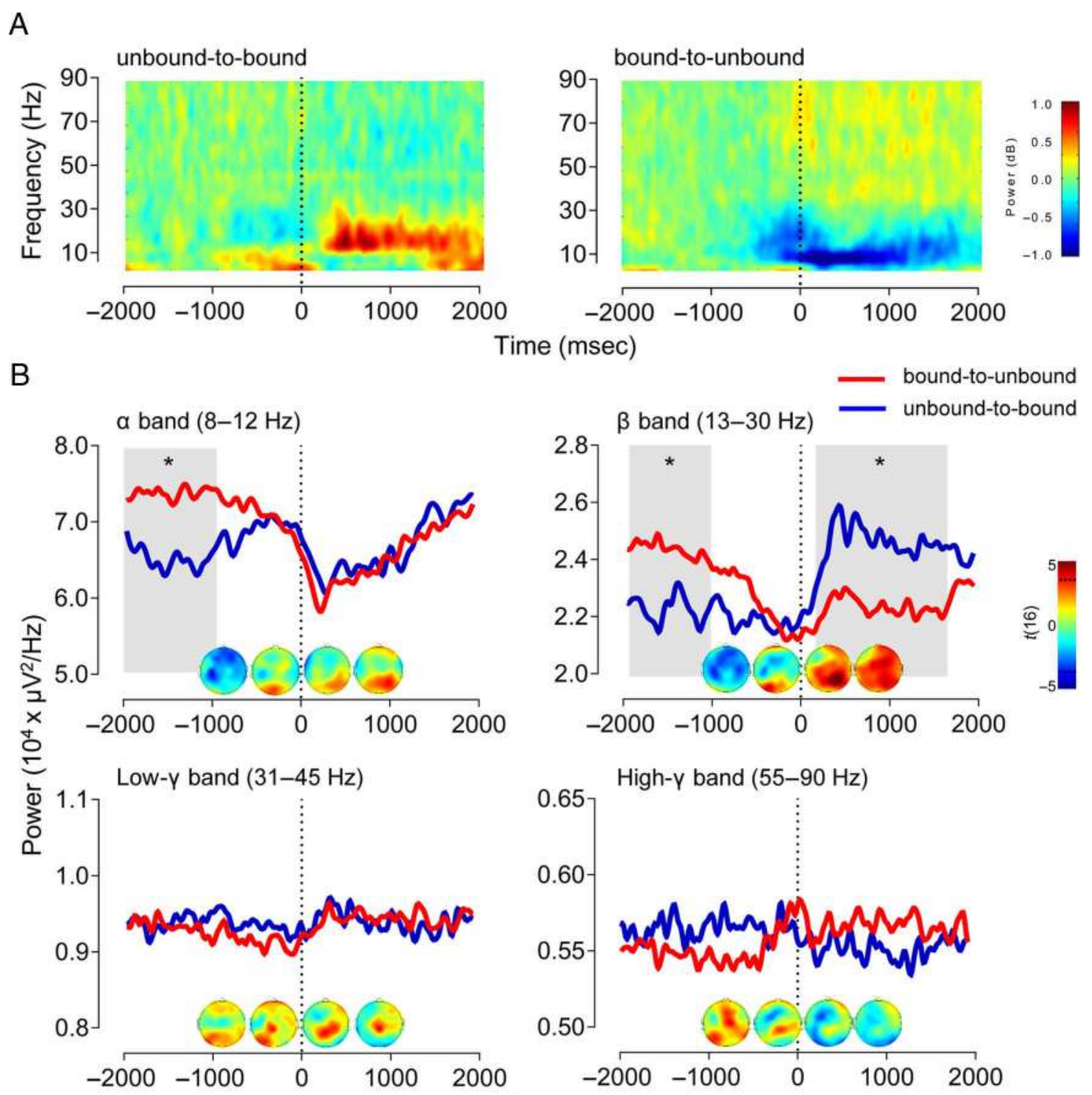

Time (msec)
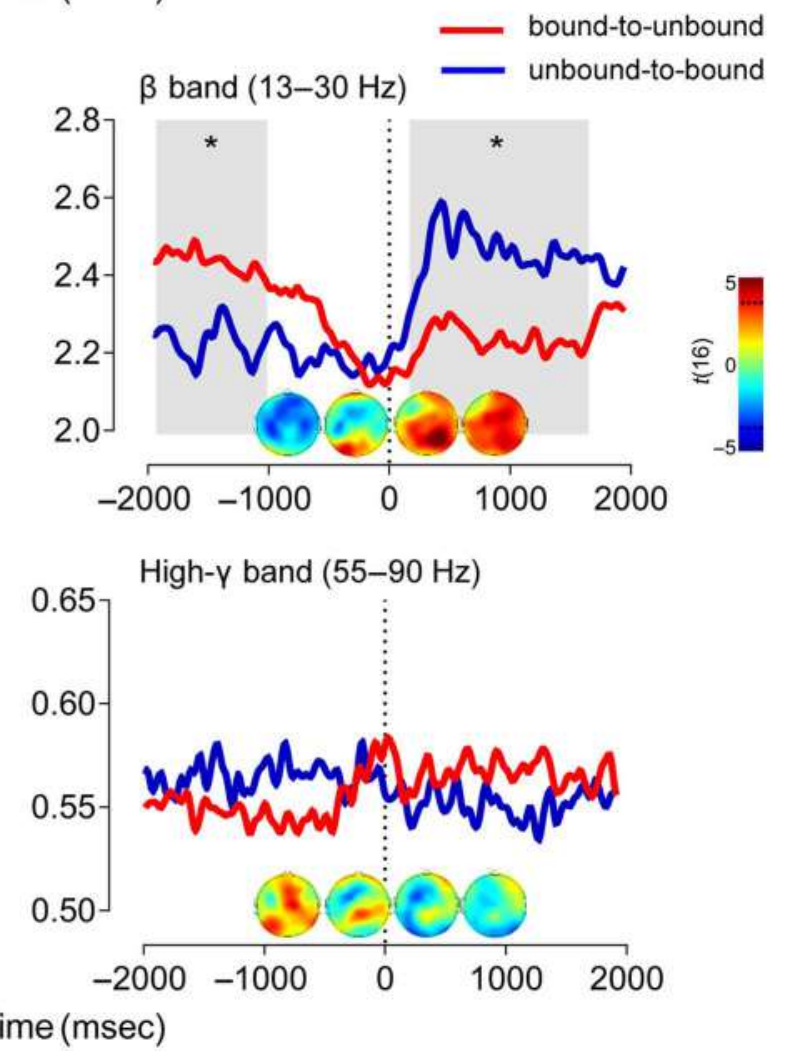

Figure 7. Time course of brain oscillations during perceptual switches. (A) Spectrograms of transitions from unbound to bound perception (left) and from bound to unbound perception (right). The zero time point represents the moment participants reported the switch. The interval from -1500 to $-1000 \mathrm{msec}$ was used as baseline for visualization purposes. (B) Curves represent the time course of average absolute power for alpha, beta, and low- and high-gamma frequency bands over all electrodes during transitions to bound (blue lines) or to unbound (red lines). Significant differences (shaded areas) in alpha power were found before perceptual switches $(-1900$ to $-900 \mathrm{msec}, p<.002)$, whereas beta oscillations show significant differences both before perceptual change $(-2000$ to $-1000 \mathrm{msec}, p<.005)$ and after it $(500-1700 \mathrm{msec}, p<.0001)$. Topographies of power difference (contrast bound vs. unbound) are shown for the time windows -1000 to $-500,-500$ to $0,0-500$, and $500-1000$ msec.

entire task, and no correlation was observed between oscillations and gaze positions or saccades (data not shown).

The amplitude of brain oscillations during periods of stable perception, measured over the whole scalp, shows differences in a range of frequencies that fall into the canonical beta band $(12-23 \mathrm{~Hz}, p<.05$ FDR corrected) between distinct perceptual states arising from viewing the ambiguous image (Figure 6A). Higher beta activity associated with the subjectively perceived bound configuration, although measurable over several regions of the scalp, was most pronounced over right parietal regions (Figure 6A, see scalp topographies). This difference in beta power was not observed for similar bound and unbound percepts elicited by the unambiguous stimulus (Figure 6B), suggesting that beta activity might be related to processes of active disambiguation.

The dynamics of oscillatory activity during periods of perceptual change, seen in the time-frequency plots in Figure $7 \mathrm{~A}$, reveal a main effect of perception on upper alpha and beta frequencies (the observed pattern reflects changes in the spectrum relative to the opposite condition). 
Figure 8. Classification of perceptual switches. (A) Accuracy of the binary classification of trials consisting of unbound-to-bound or boundto-unbound changes. Trials were classified using an SVM algorithm based on the power spectrum before $(-2000$ to 0 msec) and after (0-2000 msec) perceptual reports (85 discrete frequencies features, 2 temporal features; see Methods for details). Above-chance classification was significant for 9 of 16 participants. (B) Normalized weight vectors for discrete frequency bands for periods before (Pre) and after (Post) a perceptual report (top). Absolute weights (average of absolute Pre and Post normalized weight vectors) show a greater contribution of the beta band for discriminating perceptual transitions (bottom).

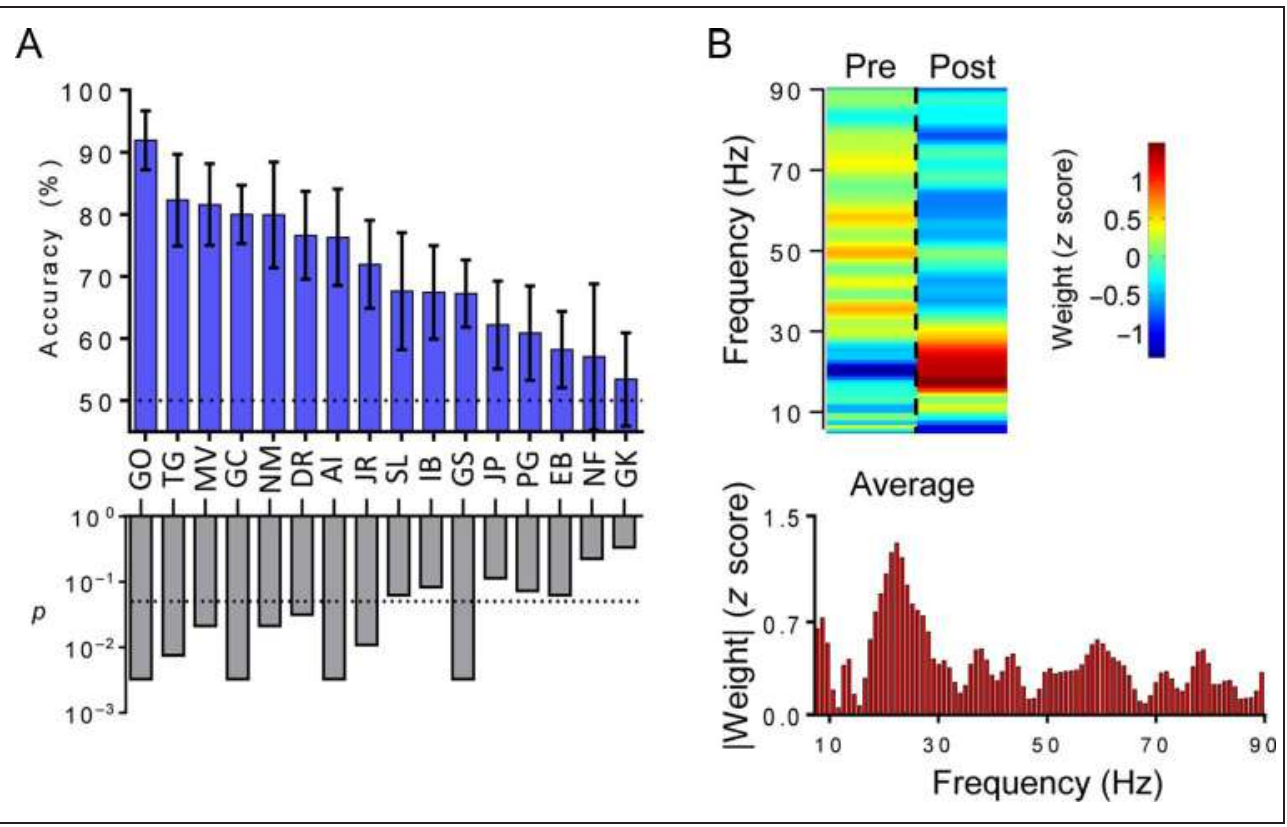

The time courses of power at distinct frequency bands in Figure $7 \mathrm{~B}$ show that most activity related to changes in perception is found in the alpha and beta frequency bands. Differences in the amplitude of alpha oscillations (8-12 Hz) were found preceding perceptual switches by almost $2 \mathrm{sec}(-1900$ to $-900 \mathrm{msec}, p<.002$, nonparametric cluster statistic) and corroborate the power increase observed during stable perception: the bound condition (represented by the period before 0 in the bound-tounbound condition; Figure 7B) being associated with increased power for frequencies of $12 \mathrm{~Hz}$ and above. Beta activity, on the other hand, shows a dynamic more revealing of a change in perception, seen by the contrast between beta power before and after a perceptual switch $(-2000$ to -1000 msec, $p<.005$; 500-1700 msec, $p<$ .0001 ; nonparametric cluster statistic). Increased power once again correlates with experiencing the bound configuration and seems to originate from right parietal areas (maximum differences at time 0-500 msec: P2, $t(16)=$ 5.32; $\mathrm{P} 4, t(16)=5.75 ; p<.0001$, paired $t$ test) and to spread from there to more posterior regions. In contrast, gamma oscillations, which are typically induced by moving stimuli and correlate with coherent motion (Aissani, Cottereau, Dumas, Paradis, \& Lorenceau, 2011; Siegel, Donner, Oostenveld, Fries, \& Engel, 2007), only show slight differences between the two percepts $(p>.05)$. The real transitions to bound or unbound percepts induced by the unambiguous stimulus do not follow the same dynamics of alpha and beta power as in the ambiguous stimulus (data not shown), and no differences were found over the time course of these two frequency bands.

Spectral changes measured over the entire scalp were sufficiently distinct as to allow perceptual transitions to be classified as either transitions to a bound configuration or to an unbound one based on power estimates. Accuracies well above chance, around $70 \%$ (mean $=$ $70.92 \%, S D=10.89 \%$, maximal accuracy of $91.92 \pm$ $4.74 \%$; Figure 8A), were obtained using an SVM classifier, which performed a binary classification of perceptual switches using power values before and after a perceptual report ( 85 frequency features and 2 time window features, to a total of 170 features). For the nine participants for which this machine learning method produced a significant classification of trials, the frequencies most relevant for discriminating perceptual switches were those around the beta band (Figure 8B).

\section{DISCUSSION}

Previous studies on the BBS theory have hypothesized that long-range synchrony is correlated with holistic perception. In a seminal study in the cat visual cortex, synchrony across hemispheres has been approached as a critical scenario to evaluate functional connectivity of distant neural assemblies and its role in binding (Engel et al., 1991). In the context of visual binding, long-range synchrony has been studied using collinear grating stimuli (Knyazeva, Fornari, Meuli, \& Maeder, 2006) and stroboscopic alternative motion (Rose \& Buchel, 2005) as well as tasks probing conscious recognition of faces (Castelhano, Duarte, Wibral, Rodriguez, \& Castelo-Branco, 2014; Rodriguez et al., 1999), familiar objects (Mima, Oluwatimilehin, Hiraoka, \& Hallett, 2001), and words (Melloni et al., 2007). These studies suggest that increased coherence is concurrent with good Gestalt configurations and correlates with holistic perception. Nonetheless, the 
BBS question has been difficult to resolve in either animal models, as previously attempted by one of the coauthors (Castelo-Branco, Goebel, Neuenschwander, \& Singer, 2000), which are limited by absent or indirect perceptual reports (Shadlen \& Movshon, 1999), or humans, as explicit and unequivocal testing for long-range synchronization had not been possible because of both recording and paradigm constraints. We believe that our study may help solve this ongoing dispute (Palanca \& DeAngelis, 2005; Roelfsema, Lamme, \& Spekreijse, 2004; Thiele \& Stoner, 2003; Treisman, 1999) by using a bistable perceptual integration paradigm that explicitly requires interhemispheric binding and therefore allows a straightforward test of the BBS hypothesis.

To circumvent the abovementioned challenges, we employed a paradigm where visual binding required interhemispheric integration under conditions of constant and physically identical sensory stimulation. The ambiguous stimulus was based on the work of Hans Wallach (1935) and resembles a classic barber pole illusion mirrored at the visual midline so as to cast two distinct images at each visual hemifield. The ambiguity arises as the image can be perceived either as a single integrated moving pattern or as two images segregated across hemifields with opposite motion directions, producing a strong perceptual correlate of binding in the former or lack thereof in the latter. The analysis of the dynamics of perception revealed that, despite a perceptual bias toward a dominant bound percept, the stimulus behaves as a typical bistable image (Borsellino, De Marco, Allazetta, Rinesi, \& Bartolini, 1972), with perceptual durations tending to follow a gamma distribution (Kline, Holcombe, \& Eagleman, 2004; Sterzer, Russ, Preibisch, \& Kleinschmidt, 2002), and denotes the presence of competing neural representations. The perceptual changes reported under ambiguous conditions are thus the result of endogenous processes, leading to an alternation of dominance between competing neural assemblies, and were explored to study electrophysiological signatures of perceptual interpretation.

We could study the BBS using the described bistable stimulus as a paradigm of perceptual binding and EEG to estimate the coupling of neural activity between distant brain regions. According to this hypothesis, the unified percept should result from the synchronous activity of separate brain areas (Engel \& Singer, 2001); specifically, in the current paradigm, such communication should occur through the corpus callosum (Genc, Bergmann, Singer, \& Kohler, 2011; Engel et al., 1991). Although events of coherent activity are usually brief, in the order of a few hundred milliseconds (Varela et al., 2001), implicit in the BBS is the prediction that stable periods of bound perception should be supported by prolonged, or at least recurrent, periods of neural synchrony. In fact, findings of persistent beta and gamma synchrony correlated to binding (Knyazeva, Fornari, Meuli, \& Maeder, 2006; Rose \& Buchel, 2005; Knyazeva et al., 1999) have been reported in support of the BBS. Moreover, noninvasive stimulation (i.e., tACS) leading to the uncoupling of brain regions engaged in the binding of visual stimuli has provided evidence beyond simple correlation (Struber et al., 2014) but that can still be confounded by effects on perceptual switch rate and not necessarily binding (Cabral-Calderin, SchmidtSamoa, \& Wilke, 2015). For this reason, we tested whether synchrony is correlated with stable perceptual binding, which, if true, would strongly argue in favor of synchrony as a substrate for binding. We found that synchrony between both hemispheres was similar for bound and unbound percepts, and this was true for both ambiguous and unambiguous stimulations. The ambiguous stimulus was intended to dissociate perceptual binding from changes in sensory input, because it is known that visual input can drive synchronous activity that may, nonetheless, be entirely unrelated to binding (Bair, 1999). It follows from the BBS then that, if binding was carried out by long-range synchrony, a bound configuration should lead to an increase in interhemispheric synchrony, which we did not observe to occur to a significant extent in both sensor and source space. An effort was made to account for both zero-lag and nonzero phase-lag phase synchrony in the present paradigm, because the BBS in its original form proposes binding to rely mainly on zero-phase lag synchrony although other forms of communication through nonzero phase delays have since been put forward (Bastos, Vezoli, \& Fries, 2015). None of the approaches indeed revealed increased interhemispheric synchrony. The source-based connectivity analysis focused on interhemispheric binding between visual brain areas, to guard against Type II errors. Significant connectivity in the beta frequency was found between regions corresponding to hMT/V5 and right posterior parietal regions. hMT/V5 is known to be involved in global motion integration, and the possibility that a study focusing on this region might confirm its role in interhemispheric binding remains open (Hipp et al., 2011). Our findings seem nevertheless to contrast with those of increased interhemispheric coherence correlated with moving patterns adhering to good Gestalt configurations reported by others (Knyazeva, Fornari, Meuli, Innocenti, \& Maeder, 2006; Knyazeva, Fornari, Meuli, \& Maeder, 2006). As discussed above, we believe that the current paradigm is ideally suited to evaluate the role of synchrony in long-range binding because binding was directly probed through perceptual judgments under constant stimulation and required across hemisphere integration of orthogonally oriented, instead of collinear, patterns. In fact, several of the studies showing a positive correlation between binding and synchrony have employed collinear moving stimuli (Knyazeva, Fornari, Meuli, \& Maeder, 2006; Knyazeva et al., 1999; Kreiter \& Singer, 1996; Engel et al., 1991; Gray \& Singer, 1989), which could generate synchrony among neurons with similar receptive fields through mechanisms unrelated to grouping (Ts'o, Gilbert, \& Wiesel, 1986) and may not generalize to scenarios where grouping occurs between neurons with noncollinear receptive fields (Palanca \& 
DeAngelis, 2005). Our design required obligatory interhemispheric (i.e., long-range) synchrony during bound percepts for the BBS to hold true and thereby overcomes the limitations of scalp recording in the study of synchrony. The fact that gamma frequencies are attenuated in human EEG compared with invasive recordings in other species (Juergens, Guettler, \& Eckhorn, 1999), a fact echoed by recent findings showing synchronous activity to be a spatially limited phenomena over cortical layers (Zandvakili \& Kohn, 2015), might suggest a reserved interpretation of the current findings, which concerns the gamma frequency band. We believe that, even in such a case, given the recent interest in exploring abnormal synchrony as a source of human cognitive impairment, the present work offers a necessary and nuanced perspective in the discussion of the role of synchrony in human cognition.

Despite an absence of change in long-range gamma synchrony related to different perceptual configurations, we did however find oscillatory activity related to distinct visual experiences, as differences in EEG power at beta frequencies were found between both percepts and beta power was clearly modulated during perceptual transitions. Importantly, moments of perceptual changes could be correctly classified using automatic methods based on these spectral differences. Although, in our current study, increased beta power was found to correlate with motion binding, it is intriguing that it was only observed under conditions of ambiguity. We suggest that this may be the case because only under ambiguity endogenous switch mechanisms dominate, and beta modulation may underlie such endogenous perceptual changes. These findings need to be reconciled with studies showing increased beta activity to be either correlated (Aissani, Martinerie, Yahia-Cherif, Paradis, \& Lorenceau, 2014) or anticorrelated (Zaretskaya \& Bartels, 2015) with visual integration/Gestalt perception. Our findings parallel those of VanRullen et al. (2006) and Piantoni, Kline, and Eagleman (2010) showing differences in beta power related to visual experience. In these studies, under stimulation with a bistable moving stimulus, namely, the Continuous Wagon Wheel Illusion, increased beta power was shown to correlate with perception of the most probable configuration, that is, the one with a higher likelihood of being perceived. In fact, the time course of alpha and beta power observed in the current study closely resembles those reported by Piantoni et al. (2010, see Figures 2 and 3), suggesting common perceptual mechanisms and/or neural substrates operating during perceptual dominance in different bistable stimuli. Because most findings in bistability show increased beta activity related to perception of the most probable conformation, usually the one perceived most of the time (Piantoni et al., 2010; VanRullen et al., 2006; this study) or the conformation first perceived in short presentations (Zaretskaya \& Bartels, 2015), we favor the interpretation that, rather than binding, beta activity is related to the internal state of one's perception and identifies the configuration that is most favored under ambigu- ous conditions. The lack of spectral differences in the unambiguous condition is in agreement with this conclusion. Hence, the increased beta activity likely corresponds to top-down modulations, revealed under ambiguity, that drive perception toward a default percept (bound, in the current case), among a set of equally valid interpretations, and temporarily dissipates when a novel one becomes dominant. This would concur with the hypothesis of beta signalling a status quo of the cognitive system (Engel \& Fries, 2010) and follows from findings of beta activity on the extrastriate cortex originating from cortical feedback, rather than being driven by inputs from V1 (Schmiedt et al., 2014).

\section{Conclusions}

Our results show that distinct patterns of oscillatory activity are associated with distinct contents of perception. These oscillatory signatures of distinct visual experience do not reside, however, on synchronous activity between distant neural assemblies in the gamma range, which, in the current study, excludes gamma synchrony as the carrier of visual binding. Future studies should explore the role beta oscillations play in visual disambiguation and whether it conveys information pertaining specifically to binding.

\section{Acknowledgments}

This work was funded by "Projecto Operacional Regional do Centro," CENTRO-01-0145-FEDER-000016; MEDPersyst, POCI01-0145-FEDER-016428; FCT, UID/NEU/04539/2013; and COMPETE, POCI-01-0145-FEDER-007440 and by the Bial Fellowship Programme 2014/15 Grant Edition G-02384, application number $373 / 2014$. J. V. D. was supported by the Portuguese Foundation for Science and Technology with the individual scholarship SFRH/BD/69735/2010.

Reprint requests should be sent to Gabriel Nascimento Costa or Miguel Castelo-Branco, Visual Neuroscience Laboratory, IBILIFaculty of Medicine, University of Coimbra, Azinhaga de Santa Comba, 3000-548 Coimbra, Portugal, or via e-mail: gcosta18@ gmail.com, mcbranco@fmed.uc.pt.

\section{REFERENCES}

Aissani, C., Cottereau, B., Dumas, G., Paradis, A. L., \& Lorenceau, J. (2011). Magnetoencephalographic signatures of visual form and motion binding. Brain Research, 1408, 27-40.

Aissani, C., Martinerie, J., Yahia-Cherif, L., Paradis, A. L., \& Lorenceau, J. (2014). Beta, but not gamma, band oscillations index visual form-motion integration. PLoS One, 9, e95541.

Bair, W. (1999). Spike timing in the mammalian visual system. Current Opinion in Neurobiology, 9, 447-453.

Bastos, A. M., Vezoli, J., \& Fries, P. (2015). Communication through coherence with inter-areal delays. Current Opinion in Neurobiology, 31, 173-180.

Benjamini, Y., \& Yekutieli, D. (2001). The control of the false discovery rate in multiple testing under dependency. Annals of Statistics, 29, 1165-1188. 
Borsellino, A., De Marco, A., Allazetta, A., Rinesi, S., \& Bartolini, B. (1972). Reversal time distribution in the perception of visual ambiguous stimuli. Kybernetik, 10, 139-144.

Brainard, D. H. (1997). The Psychophysics Toolbox. Spatial Vision, 10, 433-436.

Cabral-Calderin, Y., Schmidt-Samoa, C., \& Wilke, M. (2015). Rhythmic gamma stimulation affects bistable perception. Journal of Cognitive Neuroscience, 27, 1298-1307.

Castelhano, J., Duarte, I. C., Wibral, M., Rodriguez, E., \& Castelo-Branco, M. (2014). The dual facet of gamma oscillations: Separate visual and decision making circuits as revealed by simultaneous EEG/fMRI. Human Brain Mapping, 35, 5219-5235.

Castelo-Branco, M., Goebel, R., Neuenschwander, S., \& Singer, W. (2000). Neural synchrony correlates with surface segregation rules. Nature, 405, 685-689.

Cohen, M. X. (2014). Analyzing neural time series data: Theory and practice (1st ed.). Cambridge, MA: MIT Press.

Cortes, C., \& Vapnik, V. (1995). Support-vector networks. Machine Learning, 20, 273-297.

Delorme, A., \& Makeig, S. (2004). EEGLAB: An open source toolbox for analysis of single-trial EEG dynamics including independent component analysis. Journal of Neuroscience Methods, 134, 9-21.

Dhamala, M., Rangarajan, G., \& Ding, M. (2008). Analyzing information flow in brain networks with nonparametric Granger causality. Neuroimage, 41, 354-362.

Engel, A. K., \& Fries, P. (2010). Beta-band oscillations-Signalling the status quo? Current Opinion in Neurobiology, 20, 156-165.

Engel, A. K., Konig, P., Kreiter, A. K., \& Singer, W. (1991). Interhemispheric synchronization of oscillatory neuronal responses in cat visual cortex. Science, 252, 1177-1179.

Engel, A. K., \& Singer, W. (2001). Temporal binding and the neural correlates of sensory awareness. Trends in Cognitive Sciences, 5, 16-25.

Fries, P. (2009). Neuronal gamma-band synchronization as a fundamental process in cortical computation. Annual Review of Neuroscience, 32, 209-224.

Genc, E., Bergmann, J., Singer, W., \& Kohler, A. (2011). Interhemispheric connections shape subjective experience of bistable motion. Current Biology, 21, 1494-1499.

Goldfarb, L., \& Treisman, A. (2013). Counting multidimensional objects: Implications for the neural-synchrony theory. Psychological Science, 24, 266-271.

Gray, C. M. (1999). The temporal correlation hypothesis of visual feature integration: Still alive and well. Neuron, 24, 31-47, 111-125.

Gray, C. M., Konig, P., Engel, A. K., \& Singer, W. (1989). Oscillatory responses in cat visual cortex exhibit inter-columnar synchronization which reflects global stimulus properties. Nature, 338, 334-337.

Gray, C. M., \& Singer, W. (1989). Stimulus-specific neuronal oscillations in orientation columns of cat visual cortex. Proceedings of the National Academy of Sciences, U.S.A., 86, 1698-1702.

Gross, J., Kujala, J., Hamalainen, M., Timmermann, L., Schnitzler, A., \& Salmelin, R. (2001). Dynamic imaging of coherent sources: Studying neural interactions in the human brain. Proceedings of the National Academy of Sciences, U.S.A., 98, 694-699.

Hipp, J. F., Engel, A. K., \& Siegel, M. (2011). Oscillatory synchronization in large-scale cortical networks predicts perception. Neuron, 69, 387-396.

Histed, M. H., \& Maunsell, J. H. (2014). Cortical neural populations can guide behavior by integrating inputs linearly, independent of synchrony. Proceedings of the National Academy of Sciences, U.S.A., 111, E178-E187.
Hupe, J. M., \& Rubin, N. (2003). The dynamics of bi-stable alternation in ambiguous motion displays: A fresh look at plaids. Vision Research, 43, 531-548.

Juergens, E., Guettler, A., \& Eckhorn, R. (1999). Visual stimulation elicits locked and induced gamma oscillations in monkey intracortical- and EEG-potentials, but not in human EEG. Experimental Brain Research, 129, 247-259.

Kiani, R., Churchland, A. K., \& Shadlen, M. N. (2013). Integration of direction cues is invariant to the temporal gap between them. Journal of Neuroscience, 33, 16483-16489.

Kline, K., Holcombe, A. O., \& Eagleman, D. M. (2004). Illusory motion reversal is caused by rivalry, not by perceptual snapshots of the visual field. Vision Research, 44, 2653-2658.

Knyazeva, M. G., Fornari, E., Meuli, R., Innocenti, G., \& Maeder, P. (2006). Imaging of a synchronous neuronal assembly in the human visual brain. Neuroimage, 29, 593-604.

Knyazeva, M. G., Fornari, E., Meuli, R., \& Maeder, P. (2006). Interhemispheric integration at different spatial scales: The evidence from EEG coherence and fMRI. Journal of Neurophysiology, 96, 259-275.

Knyazeva, M. G., Kiper, D. C., Vildavski, V. Y., Despland, P. A., Maeder-Ingvar, M., \& Innocenti, G. M. (1999). Visual stimulus-dependent changes in interhemispheric EEG coherence in humans. Journal of Neurophysiology, 82, 3095-3107.

Kopell, N., Ermentrout, G. B., Whittington, M. A., \& Traub, R. D. (2000). Gamma rhythms and beta rhythms have different synchronization properties. Proceedings of the National Academy of Sciences, U.S.A., 97, 1867-1872.

Kreiter, A. K., \& Singer, W. (1996). Stimulus-dependent synchronization of neuronal responses in the visual cortex of the awake macaque monkey. Journal of Neuroscience, 16, 2381-2396.

Leopold, D. A., \& Logothetis, N. K. (1999). Multistable phenomena: Changing views in perception. Trends in Cognitive Sciences, 3, 254-264.

Lindner, M., Vicente, R., Priesemann, V., \& Wibral, M. (2011). TRENTOOL: A Matlab open source toolbox to analyse information flow in time series data with transfer entropy. BMC Neuroscience, 12, 119.

Maris, E., \& Oostenveld, R. (2007). Nonparametric statistical testing of EEG- and MEG-data. Journal of Neuroscience Methods, 164, 177-190.

Melloni, L., Molina, C., Pena, M., Torres, D., Singer, W., \& Rodriguez, E. (2007). Synchronization of neural activity across cortical areas correlates with conscious perception. Journal of Neuroscience, 27, 2858-2865.

Mima, T., Oluwatimilehin, T., Hiraoka, T., \& Hallett, M. (2001). Transient interhemispheric neuronal synchrony correlates with object recognition. Journal of Neuroscience, 21, 3942-3948.

Musall, S., von Pfostl, V., Rauch, A., Logothetis, N. K., \& Whittingstall, K. (2014). Effects of neural synchrony on surface EEG. Cerebral Cortex, 24, 1045-1053.

Nikolic, D., Fries, P., \& Singer, W. (2013). Gamma oscillations: Precise temporal coordination without a metronome. Trends in Cognitive Sciences, 17, 54-55.

Nolte, G., Bai, O., Wheaton, L., Mari, Z., Vorbach, S., \& Hallett, M. (2004). Identifying true brain interaction from EEG data using the imaginary part of coherency. Clinical Neurophysiology, 115, 2292-2307.

Ojala, M., \& Garriga, G. C. (2010). Permutation tests for studying classifier performance. Journal of Machine Learning Research, 11, 1833-1863.

Oostenveld, R., Fries, P., Maris, E., \& Schoffelen, J. M. (2011). FieldTrip: Open source software for advanced analysis of MEG, EEG, and invasive electrophysiological data. 
Computational Intelligence and Neuroscience, 2011, 156869

Palanca, B. J., \& DeAngelis, G. C. (2005). Does neuronal synchrony underlie visual feature grouping? Neuron, 46 , 333-346.

Perrin, F., Pernier, J., Bertrand, O., \& Echallier, J. F. (1989). Spherical splines for scalp potential and current density mapping. Electroencephalography and Clinical Neurophysiology, 72, 184-187.

Perrin, F., Pernier, J., Bertrand, O., \& Echallier, J. F. (1990). Corrigenda. Electroencephalography and Clinical Neurophysiology, 76, 565-566.

Piantoni, G., Kline, K. A., \& Eagleman, D. M. (2010). Beta oscillations correlate with the probability of perceiving rivalrous visual stimuli. Journal of Vision, 10, 18.

Rodriguez, E., George, N., Lachaux, J. P., Martinerie, J., Renault, B., \& Varela, F. J. (1999). Perception's shadow: Long-distance synchronization of human brain activity. Nature, 397, 430-433.

Roelfsema, P. R., Lamme, V. A., \& Spekreijse, H. (2004). Synchrony and covariation of firing rates in the primary visual cortex during contour grouping. Nature Neuroscience, 7 , 982-991.

Rose, M., \& Buchel, C. (2005). Neural coupling binds visual tokens to moving stimuli. Journal of Neuroscience, 25, 10101-10104.

Schmiedt, J. T., Maier, A., Fries, P., Saunders, R. C., Leopold, D. A., \& Schmid, M. C. (2014). Beta oscillation dynamics in extrastriate cortex after removal of primary visual cortex. Journal of Neuroscience, 34, 11857-11864.

Shadlen, M. N., \& Movshon, J. A. (1999). Synchrony unbound: A critical evaluation of the temporal binding hypothesis. Neuron, 24, 67-77, 111-125.

Siegel, M., Donner, T. H., Oostenveld, R., Fries, P., \& Engel, A. K. (2007). High-frequency activity in human visual cortex is modulated by visual motion strength. Cerebral Cortex, 17, $732-741$.

Singer, W. (2001). Consciousness and the binding problem. Annals of the New York Academy of Sciences, 929, 123-146.

Singer, W., \& Gray, C. M. (1995). Visual feature integration and the temporal correlation hypothesis. Annual Review of Neuroscience, 18, 555-586.

Smith, M. L., Gosselin, F., \& Schyns, P. G. (2006). Perceptual moments of conscious visual experience inferred from oscillatory brain activity. Proceedings of the National Academy of Sciences, U.S.A., 103, 5626-5631.
Sterzer, P., Russ, M. O., Preibisch, C., \& Kleinschmidt, A. (2002). Neural correlates of spontaneous direction reversals in ambiguous apparent visual motion. Neuroimage, 15, 908-916.

Struber, D., Rach, S., Trautmann-Lengsfeld, S. A., Engel, A. K., \& Herrmann, C. S. (2014). Antiphasic $40 \mathrm{~Hz}$ oscillatory current stimulation affects bistable motion perception. Brain Topography, 27, 158-171.

Thiele, A., \& Stoner, G. (2003). Neuronal synchrony does not correlate with motion coherence in cortical area MT. Nature, 421, 366-370.

Treisman, A. (1999). Solutions to the binding problem: Progress through controversy and convergence. Neuron, 24, 105-110, $111-125$.

Ts'o, D. Y., Gilbert, C. D., \& Wiesel, T. N. (1986). Relationships between horizontal interactions and functional architecture in cat striate cortex as revealed by cross-correlation analysis. Journal of Neuroscience, 6, 1160-1170.

Uhlhaas, P. J., Pipa, G., Lima, B., Melloni, L., Neuenschwander, S., Nikolic, D., et al. (2009). Neural synchrony in cortical networks: History, concept and current status. Frontiers in Integrative Neuroscience, 3, 17.

VanRullen, R., Reddy, L., \& Koch, C. (2006). The continuous wagon wheel illusion is associated with changes in electroencephalogram power at approximately $13 \mathrm{~Hz}$. Journal of Neuroscience, 26, 502-507.

Varela, F., Lachaux, J. P., Rodriguez, E., \& Martinerie, J. (2001). The brainweb: Phase synchronization and large-scale integration. Nature Reviews Neuroscience, 2, 229-239.

Von der Malsburg, C. (1981). The correlation theory of brain function. Internal report, 81-82. Göttingen, Germany: Max Planck Institute for Biophysical Chemistry.

Wallach, H. (1935). Uber visuell wahrgenommene Bewegungsrichtung. Psychologische Forschung, 20, 325-380.

Wang, L., Mruczek, R. E., Arcaro, M. J., \& Kastner, S. (2015). Probabilistic maps of visual topography in human cortex. Cerebral Cortex, 25, 3911-3931.

Wuerger, S., Shapley, R., \& Rubin, N. (1996). "On the visually perceived direction of motion" by Hans Wallach: 60 Years later. Perception, 25, 1317-1318.

Zandvakili, A., \& Kohn, A. (2015). Coordinated neuronal activity enhances corticocortical communication. Neuron, 87 , 827-839.

Zaretskaya, N., \& Bartels, A. (2015). Gestalt perception is associated with reduced parietal beta oscillations. Neuroimage, 112, 61-69. 